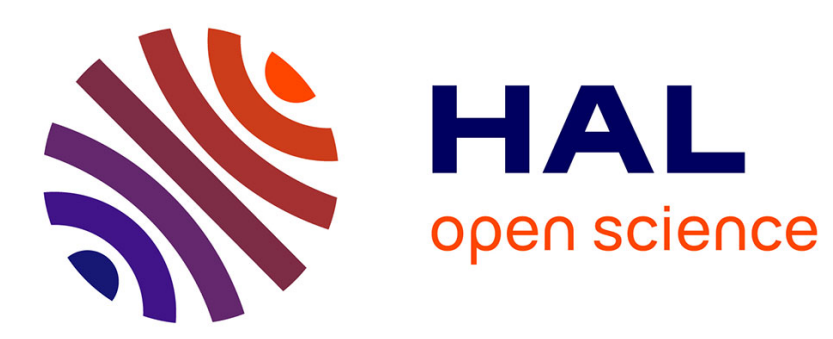

\title{
Micromechanical modeling of damage and load transfer in particulate composites with partially debonded interface
}

Nicolas Despringre, Yves Chemisky, Kevin Bonnay, Fodil Meraghni

\section{- To cite this version:}

Nicolas Despringre, Yves Chemisky, Kevin Bonnay, Fodil Meraghni. Micromechanical modeling of damage and load transfer in particulate composites with partially debonded interface. Composite Structures, 2016, 155, pp.77-88. 10.1016/j.compstruct.2016.06.075 . hal-02536455

\section{HAL Id: hal-02536455 \\ https://hal.science/hal-02536455}

Submitted on 8 Apr 2020

HAL is a multi-disciplinary open access archive for the deposit and dissemination of scientific research documents, whether they are published or not. The documents may come from teaching and research institutions in France or abroad, or from public or private research centers.
L'archive ouverte pluridisciplinaire HAL, est destinée au dépôt et à la diffusion de documents scientifiques de niveau recherche, publiés ou non, émanant des établissements d'enseignement et de recherche français ou étrangers, des laboratoires publics ou privés. 


\title{
Micromechanical modeling of damage and load transfer in particulate composites with partially debonded interface
}

\author{
Nicolas Despringre, Yves Chemisky, Kevin Bonnay, Fodil Meraghni* \\ Arts et Métiers ParisTech Metz, LEM3 UMR CNRS 7239, ENSAM Campus de Metz, 4 rue Augustin Fresnel, 57078 Metz, France
}

\begin{abstract}
A B S T R A C T
A new micromechanical damage model accounting for progressive interface debonding is developed for composite materials. It consists of an original evolution law of the damage at the interface and an appropriate load transfer law at the matrix-fiber interface integrated into a generalized incremental MoriTanaka homogenization scheme. The interface damage evolution is driven by the interfacial stress state while the load transfer is obtained from a new model inspired by the shear lag model. Specifically, such damage evolution is supported by experimental microscopic observations for short glass fiber reinforced polyamide-66.

The proposed model is validated based on numerical reference solutions provided from finite element analyses of a representative unit cell of a composite, where imperfect interfaces are represented using cohesive elements. A further comparison with experimental data proves that the proposed model is an alternative to micromechanical models involving weak interfaces in the case of spherical reinforcements. It is shown that the proposed model is able to accurately reproduce the non-linear effective response of composite materials for a broad range of reinforcement shapes, including spherical particles and matrix mechanical properties.
\end{abstract}

\section{Introduction}

Composite materials have received increasing attention in the past forty years thanks to their excellent weight to mechanical properties ratio. Specifically, short fiber reinforced composites (SFRC) have been considered as a good alternative to metals, especially in the automotive industry, to reduce gas emission through the reduction of automotive vehicle mass. Their high thermomechanical performance to density ratio allows for the design of lightweight structural parts. However, the microstructure of such materials, combined with the matrix sensitivity to environmental conditions, has a strong impact on their overall behavior and specifically on the apparition of damage. Damage in SFRC occurs at the microscopic level according to different physical degradation mechanisms, namely: interfacial decohesion, fiber breakage and matrix microcracks [1-5]. All these studies have shown that fiber/ matrix interfacial debonding is the predominant damage mechanism and plays a crucial role in the progressive degradation of the effective behavior.
The development of predictive models for fatigue of composites requires proper descriptions of these phenomena, which depends on local mechanical fields. Micromechanics therefore appears to be an adapted approach to combine the required the knowledge of the local stress state in each phase constituting the particulate composite and the description of the effective behavior. This type of modeling approach remain a challenge, especially considering the non-linear behavior of the matrix combined with damage. Multiscale models have always been a key approach to approximate the macroscopic behaviour of microstructure-dependent particulate composite materials such as SFRC [6,7]. The evolution of the defects and void density are therefore computed as a function of the local stress state $[8,9]$. However, the integration of damage at the interface does not properly include the evolution of the load transfer between the reinforcements and the matrix, and has to be enhanced accordingly.

Damage at the interface in composite materials has received a lot of interest in the past two decades. One of the developed methods consists of using a dedicated fiber coating also called an interface [10]. The main drawback is that such a three phase model implies that the knowledge of coating properties, which is rarely available. Hashin introduced the imperfect interface approach which accounts for the displacement and stress jump at the fiber/ matrix interface $[11,12]$. The aim was to replace the explicit three 
phase problem consisting of two constituents and an interface by a two phase homogenization with one imperfect interface. Many authors have investigated the imperfectly bonded interface $[13,14]$. Zhong and Meguid developed a new solution for the eigenstrain problem, as defined by Eshelby [15], of a spherical inclusion with an imperfect interface [16]. In addition, the shear lag model (SLM) has been developed to model the behavior of fiber reinforced composites. This approach predicts the elastic behavior of a two phase composite, whose fibers are stiffer than the matrix. It specifically gives the stress state inside the fiber. This method was originally developed by [17]. One of the main assumption of this method is that no slip occurs on the fiber/matrix interface. Recently, Jain et al. [18] have developed an equivalent debonded inclusion model for Eshelby based approaches coupled with the Cox's predictions applied for six loading cases, namley three uniaxial tensile and three shear loads.

The presence of stiff fibers induces a stress and strain distribution within the composite while the fibers bear most of the macroscopic stress, the strain in the matrix is more significant. As a consequence, this leads to a shear stress at the fiber/matrix interface, which governs the load and stress transfer between the matrix and the fibers. The definition of this stress transfer depends on the properties of the two-phase composite. Key equations will be briefly recalled in this paper. The whole method is detailed in terms of mechanical and mathematical analyses by [19,20], which are considered among the reference works in this topic. Jiang and Gao have also studied the stress transfer from matrix to fiber in short carbon fiber aluminium-matrix composites at several different thermal conditions [21]. In particular, they have compared several theories including, the shear lag model, and have considered a wide range of fiber aspect ratios.

The approach developed in this paper combines the result of an interfacial damage law with a specific load transfer model inspired by the shear lag model. Indeed, the shear lag model is utilized to determine the stress distribution of a partially debonded fiber, considering a non-zero shear stress field only at the non-damaged interface. The determination of the evolution of debonded zones depends on an evolution equation for interfacial damage, based on a probabilistic criterion. Translated into a debonded area, a shear lag model approach allows for the determination of the stress state in the fiber to be compared with the non-damaged stress state. Thus, a load transfer ratio is obtained, which is integrated into an adapted micromechanical homogenization scheme using an appropriate computation of the concentration tensor in order to determine the stress fields of the different phases. The developed approach is therefore designed to suit an incremental multiscale model and can accurately capture the nonlinear behavior accounting for progressive interface degradation.

The organization of this work is as follows. Section 2 further describes the experimental observations of interfacial damage that led to the proposition of the present load transfer model. Section 3 presents the formulation of the damage evolution law based on a statistical local criterion and the load transfer model. The end of Section 3 is devoted to the integration of such formulation into a homogenization scheme, namely the modified Mori-Tanaka model applied for particulate composites [22,8]. A numerical validation of this new approach is performed in Section 4, based on a finite element solution for interface decohesion using an advanced cohesive element approach proposed by [23]. The comparison considers the case of a short fiber reinforced polyamide 66 composite. In Section 5, the limiting case of spherical inclusions is investigated and the developed model is then applied for three types of composite with spherical reinforcements and compared with several results from literature and reference solutions: experimental results of [24,25] and a model developed by [26] for slightly weakened interfaces. The influence of the fiber aspect ratio is briefly discussed. The last section of this paper provides a conclusion summarizing the main results.

\section{Experimental observation of interface damage evolution}

Interfacial damage mechanisms have been observed by several authors in many different systems. For the particular case of short glass fiber reinforced polyamide-66, in situ damage mechanisms characterization under quasistatic monotonic loading were investigated by $[1-3,5]$. They reported that in most cases, interfacial damage starts at the fiber ends and further propagates along the fiber-matrix interface.

Following the analysis of Horst and Spoormaker [3], Arif et al. [4] have proposed a damage progression scenario where the dependance on the relative humidity $(\mathrm{RH})$ is taken into account:

- The damage starts at the fiber ends, or in areas where local stress concentration is the highest, and at locations where fibers are close to each other (all studied RH contents), as shown in Fig. 1. Specifically, for $\mathrm{RH}=0 \%$, fiber breakage occurs in addition to the previous forms of mechanisms.

- Damage interface propagates along the fiber in the form of fiber/matrix interfacial debonding as observed by Arif et al. [4] for all studied RH contents. The interfacial decohesion is accompanied by fiber breakage occurrence for $\mathrm{RH}=0 \%$ whereas it appears with a locally strained zone around the fiber for $\mathrm{RH}=50 \%$ and $100 \%$.

- Matrix microcracks develop and propagate in a brittle way for $\mathrm{RH}=0 \%$ and in a ductile way for $\mathrm{RH}=50 \%$ and $100 \%$, accompanied with high matrix deformation bands for $\mathrm{RH}=100 \%$.

- The propagation of the matrix microcracks brings about damage accumulation leading to total failure.

Such interfacial decohesion appears in PA66-GF30 along the fiber interface, shown in Fig. 1, from [4]. Fig. 1a shows that the damage along the interface leads to a rather important interfacial decohesion. The stress distribution in the phases is then strongly impacted according to the creation of free surfaces in the material. Fig. 1b illustrates that fiber ends are a principal spot for the initiation of damage.

Such observations lead to the conclusion that micromechanical predictive models with damage should include the effect of interfacial debonding. Since damage evolution is driven by the local damage state for all mechanisms, it is crucial to determine the stress distribution in the different phases where interfacial debonding propagates. In particular, the stress transfer at the damaged interface requires thorough attention in order to determine the stress state of the fibers, which impacts the overall stress distribution.

The recent development of microcomputer tomography (microCT) has pushed forward the quantitative evolution of damage propagation in composite materials [27]. The specific case of damage evolution in PA66-GF30 fatigued samples has been studied by [1]. The main features of defects, such as volume, orientation and shape, have been obtained for several levels of overall damage, represented in terms of number of cycles relative to the number of cycles to failure. One of the main conclusion is that the orientation of defects follows the orientation of fibers, which indicate that interfacial debonding is the leading damage mechanism. Indeed, the volume of defects oriented in the fiber direction is increasing throughout the lifetime of the composite. Tt is difficult to directly relate those quantifications to an evolution law of the interface decohesion surface, however, this clearly shows that such an evolution equation drives the fatigue behavior of the composite. 


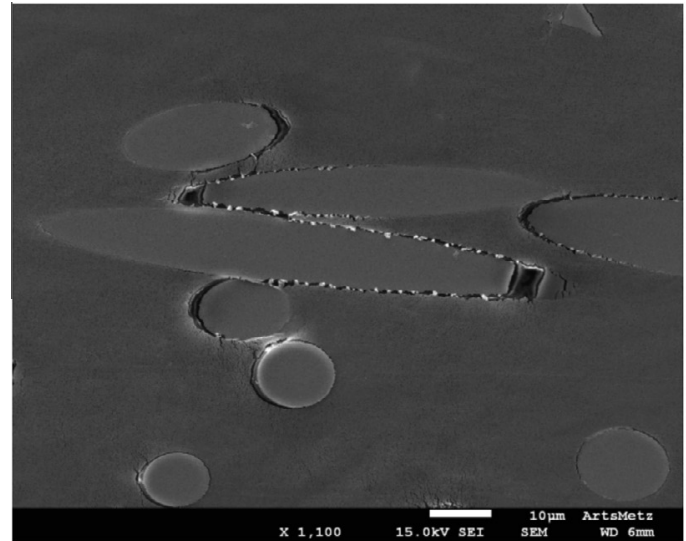

(a)

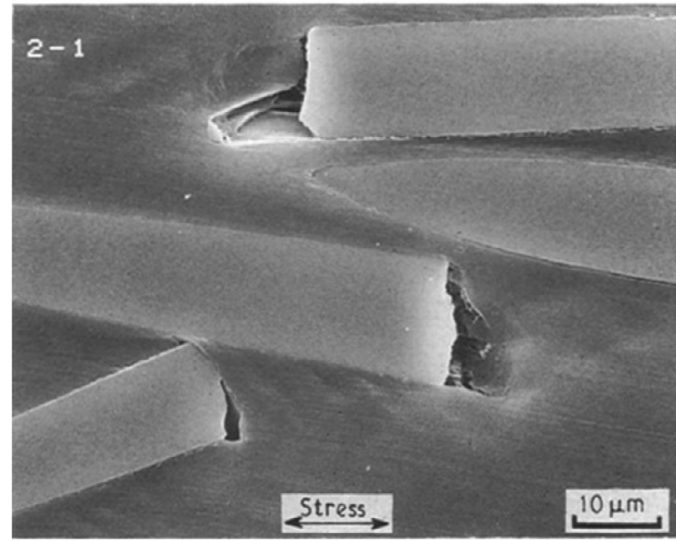

(b)

Fig. 1. SEM observations of the interfacial debonding of PA66GF30 obtained by (a) Arif et al. [1] which confirm those from Sato et al. [2] (b).

\section{Fiber/matrix interface decohesion statistical damage model}

Following the experimental observations summarized in the previous section, the proposed interfacial decohesion model consists of three components:

1. An evolution law for the damage at the interface.

2. A load transfer model.

3. An homogenization strategy.

The definition of each component of this model is independent, as long as they follow the next requirements: (i) the description of the damage for one interface is described by an internal variable, which can be scalar or tensorial - such a description can be generalized to a family of interfaces and incorporated into a probability approach; (ii) the load transfer model is written in terms of the internal variable chosen for the evolution of damage of an interface or a family of interfaces; (iii) the reinforcements are considered ellipsoidal. Note that such requirements are not mandatory, but they allow the utilization of several different homogenization methods and the facilitation of the determination of local fields that can be incorporated into the damage evolution equation or the load transfer model.

\subsection{Damage evolution law}

The evolution equation for local damage $d_{l o c}$ at one point of the interface should take into account three main feature: (i) it should depend on the local stress field at the interface (or the stress field just inside and just outside of the reinforcements); (ii) it should depend on the level of global damage $d$ of the whole interface; (iii) since one evolution equation hold for fibers with slightly different local arrangements, orientation and shape, it should be written in terms of a probability of damage $\widetilde{P}_{r}$ over a certain characteristic time $\lambda$. Such an evolution equation will have the following general form:

$\dot{d}_{l o c}=g(d) \frac{\widetilde{P}_{r}\left(\boldsymbol{\sigma}^{\text {in }}, \boldsymbol{\sigma}^{\text {out }}\right)}{\lambda}$

Such damage probability (Eq. (6)) is utilized to compute the damage increment within each time step. Global damage $d$ at the fiber-matrix interface varies between 0 (perfect interface) and 1 (full decohesion), and is computed from local damage at the interface. The influence of the local stress state is taken according to a quadratic rupture criterion (Eq. (2)). This approach is partially based on the work done by Jendli et al. [28]. This criterion depends on the interfacial stress at the interface: the normal component $\sigma_{n}$ and the shear component $\tau$. $\sigma_{\text {crit }}$ and $\tau_{\text {crit }}$ are the maximum normal and shear stress prior to full interfacial debonding, respectively. Meraghni et al. [29] describe how to obtain the normal and shear components of stress at the interface using the continuity of the traction vector at the interface. The shear and normal stress components can thus be computed at each point of the ellipsoid surface. The original form of such quadratic failure criterion is expressed as:

$\left(\frac{\sigma_{n}}{\sigma_{\text {crit }}}\right)^{2}+\left(\frac{\tau}{\tau_{\text {crit }}}\right)^{2} \leqslant 1$

To represent the differences in the stress threshold that correspond to the damage apparition for an isolated fiber and to the damage present in all fibers of the same family, a probabilistic factor $\eta$ is introduced in the criterion, which becomes $\xi\left(\sigma_{n}, \tau\right)$ according to Eq. (3).

$\xi\left(\sigma_{n}, \tau\right)=\left(\frac{\sigma_{n}}{\eta \sigma_{\text {crit }}}\right)^{2}+\left(\frac{\tau}{\eta \tau_{\text {crit }}}\right)^{2} \leqslant 1$

Furthermore, since the local arrangement of surrounding fibers introduces a variability which is, for instance, difficult to describe with mean-field homogenization techniques, a probabilistic approach aims tp describe the damage evolution in a family of reinforcements that share some common features, for example, orientation and aspect ratio. The damage evolution equation therefore accounts for the local differences in terms of stress and strain fields.

The probabilistic formulation of the criterion (Eq. (3)) is introduced using a normal distribution law in Eq. (4). It allows for the determination of the probability of rupture $P_{r}$ at the interface for any kind of interfacial stress. $\mathcal{N}$ is the cumulative distribution function of the normal law. The formulation requires three parameters: $\gamma$ (shape function parameter), $\kappa$ (damage threshold) and $\alpha$ (damage location exponent). The second parameter takes on values between 0 and 1 and governs the mean value $m$ and the standard deviation $s d$ of the normal distribution. This damage law is designed to be applied at all points of the interface since the damage evolution/propagation strongly depends on the location of the interfacial debonding.

The damage evolution is considered to be correlated with the local curvature of the envelope. This is taken into account by means of the gaussian curvature $C(\boldsymbol{x})$ of the considered surface point $\boldsymbol{x}$, relative to the gaussian curvature along the central equator of the short fiber $C_{0}(\boldsymbol{x})$ and an exponent parameter $\alpha$. 
$P_{r}\left(\sigma_{n}, \tau\right)=\mathcal{N}\left(\left(C_{0} / C\right)^{\alpha} \xi^{\gamma}, m, s d\right)$

The mean value $m$ and the standard deviation $s d$ are defined as:

$m=\frac{1+\kappa}{2} \quad s d=s d_{0}(1-\kappa)$,

where $\kappa$ adjusts the mean and standard deviation of the normal law to obtain a value of $P_{r}\left(\sigma_{N}, \tau\right)=99 \%$ along the rupture criterion. $s d_{0}$ is the standard deviation which allows for reaching a damage level of $99 \%$ for $\kappa=0$, when the failure criterion is reached.

The density function associated with normal distributions has strictly positive values on $]-\infty ;+\infty[$. Thanks to the choosen adaptive standard deviation $(s d)$, the cumulative distribution function already reaches 0.99 on $[1 ;+\infty[$. However, the density function corresponding to $]-\infty ; \kappa]$ should be zero. To this aim, the value of the density function at the abscissa $\kappa$ is subtracted from Eq. (4). Then, the equation is normalized to guarantee that the integral of the density function over the whole interval ] $-\infty ;+\infty[$ will be equal to 1 . This leads to Eq. (6):

$\tilde{P}_{r}\left(\sigma_{n}, \tau\right)=\frac{\mathcal{N}\left(\left(C_{0} / C\right)^{\alpha} \xi^{\gamma}, m, s d\right)-\mathcal{N}(\kappa, m, s d)}{1-\mathcal{N}(\kappa, m, s d)}$

Finally, the evolution equation of the local damage for each material point at the reinforcement/matrix interface, that accounts for the actual damage $d$, representative of the damage for the whole interface, and $\omega$, which is a parameter expressing the effect of the accumulated damage on its own evolution, is as follows:

$\dot{d}_{l o c}=\frac{(1-d)^{\omega}}{\lambda} * \tilde{P}_{r}\left(\sigma_{n}, \tau\right)$

Fig. 2 illustrates this last equation and shows the influence of the normal and shear components of the traction vector on damage rate at a material point of the reinforcement/matrix interface.

Since this local damage evolution equation is suitable for all material points at the interface, it can be computed over the entire envelope of the reinforcement. To this aim, two integration angles $u$ and $v$ are used to express the ellipsoid surface of the inclusion. It is worth mentioning that $u$ is the angle between the vector $x$ and the projection of the normal vector in the $x 0 y$ plane, while $v$ is the angle between the vector $z$ and the projection of the normal vector in the $x 0 z$ plane. The damage rate is then computed at each mesh point, using the corresponding normal vector. Fig. 3 show the ellipsoidal fiber with the criterion $\xi$ computed accordingly, and a damage map using the two integration angles $u$ and $v$ (both in longitudinal-transverse planes).

It has been found that the evolution of the local damage criterion around this ellipsoid is maximal around the fiber ends for tensile loading (Fig. 3a). This is consistent with the experimental

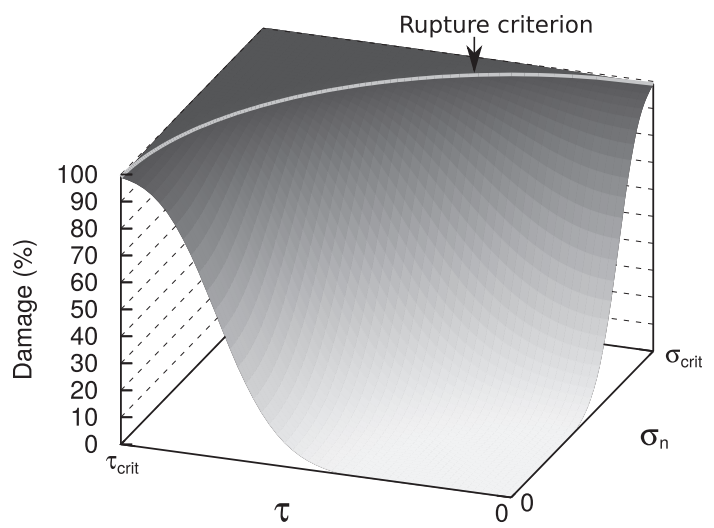

Fig. 2. Iso-damaged surfaces given by the interfacial damage law (with $\gamma=0.8$, $\kappa=0.8, \omega=1, \eta=1$ and $\lambda=1$ ). observation that damage is mostly initiated in those areas [1-5]. However, the other stress state exhibits a maximal rate in other areas of the reinforcement envelope, as seen in Fig. $3 \mathrm{~b}$. The damage computation should not be restricted to the fiber ends only, even though it appears to be the most frequent case. In the proposed model, the local damage criterion is computed over the whole envelope using an appropriate surface mesh. The maximal damage appear to be the driver of the damage evolution variable for the entire ellipsoid, since it directly depends on maximal traction (in terms of the quadratic criteria). The maximal local damage rate is therefore selected as the representative damage rate of the whole interface:

$\dot{d}=\max \left(\dot{d}_{l o c}\right)$

In the next section, the load transfer ratio is defined based on the damage at interface $d$, assuming that the damage initiates at the fiber ends. Such a unique damage variable for a family of fibers with the same orientation is convenient for the integration of this type of approach in multi-scale homogenization methods, and the localization of damage initiation is the core assumption of the load transfer model adopted.

\subsection{Load transfer model}

The presence of degraded interfaces influences the stress distribution inside the composite material. Compared to the perfectly bonded case, it is convenient to define a stress transfer problem for a partially debonded interface, which corresponds to the approach of the shear lag model as utilized by [20] on long cylindrical fibers.

Such an approach itself is not suitable in a homogenization scheme, since it does not give the complete stress state of the components, but rather provides only the uniaxial stress state of the fibers. However, the comparison between the uniaxial stress state predicted by this approach for a perfectly bonded interface and a partially debonded interface near the fiber ends can provide insight into how the stress state of the fiber is actually affected. This information can be further utilized to estimate the stress state of a partially debonded fiber based on other homogenization schemes, for instance, a Mori-Tanaka incremental scheme. The theoretical undamaged stress inside a fiber that is considered to be infinite $\sigma_{f \infty}$ is assumed to be determined from such a homogenization method. Indeed, for short fiber reinforced composites, since the aspect ratio is still rather significant (at least $\approx 10$ ), the reinforcement can be considered both as an ellipsoid for the homogenization method and as an infinite fiber from the point of view of the shear lag model. A similar technique has been adopted by Jain et al. [18] in their relevant work devoted to modeling partially debonded interfaces.

A brief review of the shear lag model (SLM) based on [20] is first presented here, which applies to cylindrical fibers. The SLM derives Eq. (9), defining the stress of a fiber section by its coordinate $z$ (origin at the fiber center). $\beta$ is the shear lag parameter as defined by McCartney [30]; $\left\langle\sigma_{f}\right\rangle$ is the average fiber stress and $\left\langle\sigma_{f \infty}\right\rangle$ is the average theoretical fiber stress if the fiber was an infinite cylinder (superscripts 1 and 2 stand for the fiber and its coating, respectively, subscripts $r$ for the fiber radius, $E_{A}$ and $G_{A}$ for the axial tensile and shear moduli and $V_{f}$ are volume fractions).

$$
\begin{aligned}
\frac{\partial^{2}\left\langle\sigma_{f}\right\rangle}{\partial z^{2}}-\beta^{2}\left\langle\sigma_{f}\right\rangle & =-\beta^{2}\left\langle\sigma_{f \infty}\right\rangle \text { where } \beta^{2} \\
& =\frac{2}{r_{1}^{2} E_{A}^{(1)} E_{A}^{(2)}}\left[\frac{E_{A}^{(1)} V_{f}^{(1)} E_{A}^{(2)} V_{f}^{(2)}}{\frac{V_{f}^{(2)}}{4 G_{A}^{(1)}}+\frac{1}{2 G_{A}^{(2)}}\left(\frac{1}{V_{f}^{(2)}} \ln \frac{1}{V_{f}^{(1)}}-1-\frac{V_{f}^{(2)}}{2}\right)}\right]
\end{aligned}
$$



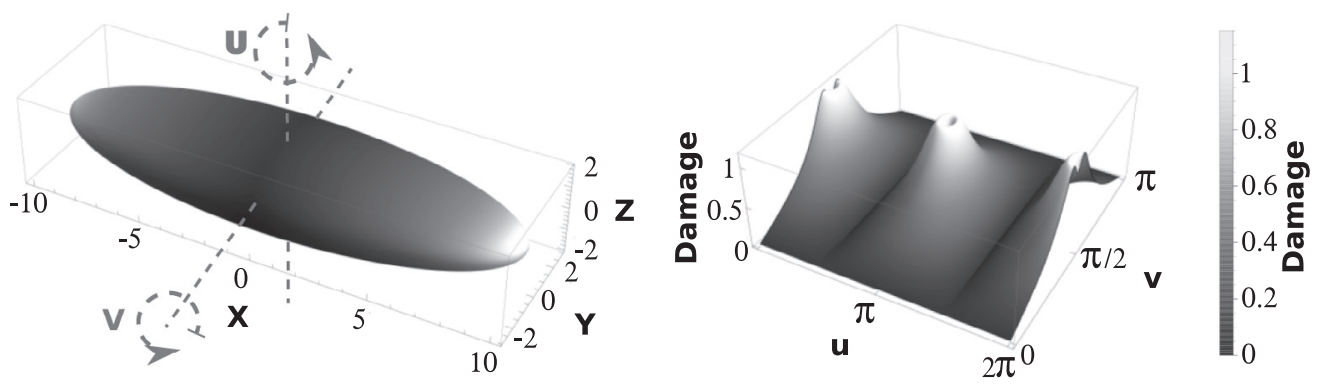

(a) Under tensile.
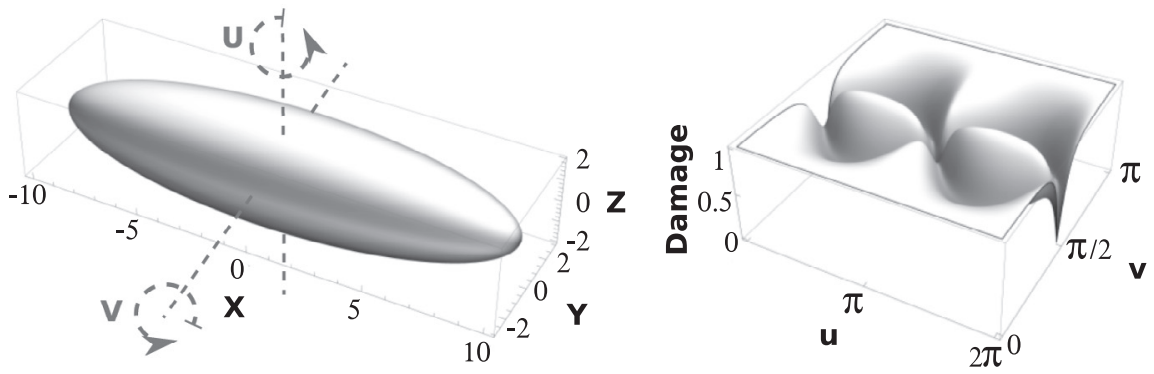

(b) Under transverse-transverse shear.

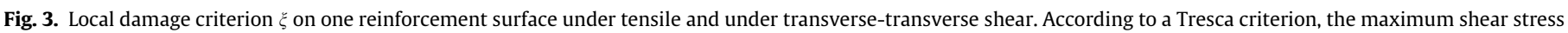
$\tau_{\text {crit }}=\sigma_{\text {crit }} / 2$, and $\eta$ is equal to 1 . The graph is a damage map using the two orientation angles $u$ and $v$ in the planes $x 0 y$ and $x 0 z$, respectively.

This differential equation is then integrated assuming, for the boundary conditions, that there is no stress at the fiber ends (Eq. (10)). In this equation, $l$ is the length of the fiber. This corresponds to debonding at the fiber ends and is appropriate with the damage scenari in short fiber reinforced thermoplastics $[1,4]$. In fact, this equation defines the load transfer ratio for each fiber section ranging from its middle to its end. Eq. (10) is at last integrated over the whole fiber to get a load transfer ratio $\Psi_{0}$ between the coating and the fiber (Eq. (11)).

$\frac{\left\langle\sigma_{f}\right\rangle(z)}{\left\langle\sigma_{f \infty}\right\rangle}=1-\frac{\cosh (\beta z)}{\cosh \left(\beta \frac{l}{2}\right)}$

$\Psi_{0}=1-\frac{2 \tanh \left(\frac{l}{2} \beta\right)}{l \beta}$

The damage parameter is then used to decrease the effective fiber length regarding load transfer, which is, in fact, reduced by the damage percent. The stress transfer ratio (Eq. (10)) is illustrated in Fig. 4a and the global load transfer ratio (Eq. (12)) in Fig. 4b.

$\Psi=\left(1-\frac{2 \tanh \left(\frac{l(1-d)}{2} \beta\right)}{l(1-d) \beta}\right)$

Due to the boundary conditions at the end of the fibers, the load transfer ratio is not equal to 1 , in terms of its bounded length, when the fiber is considered to be totally bounded. Such a ratio is therefore taken in comparison with the initial load transfer ratio in order to obtain the ratio $\chi$ to be utilized in the further homogenization method and to ensure that $\chi=1$ in a virgin material, leading to the final formula (Eq. (13)).

$\chi=\frac{\Psi}{\Psi_{0}}$

Fig. 4a shows the evolution of the local stress transfer along the length of the fiber. This shows the effect of reducing the effective length of the bounding area. In Fig. $4 \mathrm{~b}$, the global load transfer is represented as a function of the interfacial damage. First slightly

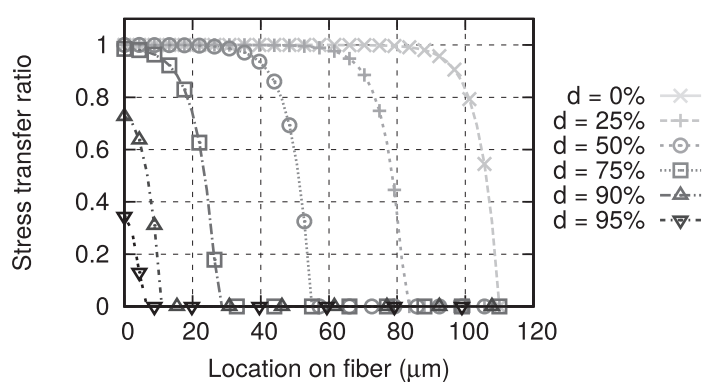

(a)

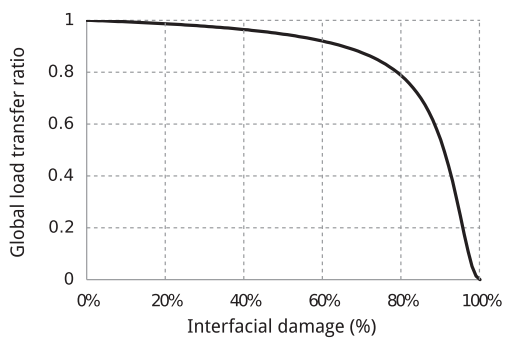

(b)

Fig. 4. Shear Lag Model (SLM) based load transfer ratio $\chi$ computed according to Eq. (13) for $220 \mu \mathrm{m}$ fiber length: (a) In each fiber section from the middle of the fiber ( 0 ) to its end, (b) for the entire fiber after normalization and as a function of interface damage.

affected with damage, when some critical value is reached (at $\mathrm{d}=75 \%$ on Fig. $4 \mathrm{a}$ for instance), the load transfer begins to drastically decrease. The load transfer eventually reaches $0 \%$ when the whole fiber interface is debonded.

\subsection{Adopted homogenization scheme}

Micromechanic models of non-linear heterogeneous materials require incrementally solving a repetitive series of steps, given an increment of strain/stress. In what follows, the methodology 
is applied by taking into account an given increment of strain. Note that other constraint drivers can be utilized (for instance, stress) by considering an additional step predicting the appropriate strain increment based on constraint drivers and the tangent modulus of the effective material.

1. The average strain increment $\Delta \overline{\boldsymbol{\varepsilon}}$ is utilized to determine the local strain increment in all the composite phases. The strain increment of the $i$-th phase is computed from the localization equation, based on a strain concentration tensor $\mathcal{A}^{i}$. Such an incremental localization relation (in which the strain concentration tensor is instantaneous and incorporates nonlinear effects [22]) of a phase has the form:

$$
\Delta \overline{\boldsymbol{\varepsilon}}^{i}=\mathcal{A}^{i} \Delta \overline{\boldsymbol{\varepsilon}}
$$

The determination of the instantaneous strain concentration tensors in the previous localization relations is a major step in the homogenization problem. Several methods exist where the composite is usually discretized into a finite number of phases with specified responses, properties and orientations. A given phase is embedded into an infinite matrix and strain concentration tensors are determined via the solution of boundary value problems [15,31]. The Mori-Tanaka Method [32,33,31,34] actually considers that the inhomogeneity is embedded in the matrix phase with the far-field conditions being that of the matrix average. Note that the determination of the instantaneous concentration tensor depends on the tangent modulus of the phases ([35]) which, in turn, depends on the strain state of the phases and thus on the localization tensors. An iterative process is therefore required in this step, where the constitutive response of the phases are computed using a trial strain increment, and where the localization tensors are corrected according to the new tangent modulus obtained.

2. The second step is the homogenization process. Knowing all the instantaneous responses of all the phases (namely the tangent modulus and the actual stress), and having determined the concentration tensor for all the phases, the global mechanical response of the composite material is obtained by:

$\overline{\mathcal{L}}=\sum_{i=0}^{N} c_{i} \mathcal{L}^{i}: \mathcal{A}^{i}$

$\overline{\boldsymbol{\sigma}}=\sum_{i=0}^{N} c_{i} \boldsymbol{\sigma}^{i}$

In the previous equations, $c_{i}$ is the volume fraction of the $i$-th phase. Furthermore, the stress transfer ratio is integrated into the homogenization step, so that the stress in the family of fibers $i$ is considered to be:

$\boldsymbol{\sigma}^{i}=\chi^{i} \boldsymbol{\sigma}_{\infty}^{i}$.

$\boldsymbol{\sigma}_{\infty}^{i}$ correspond to the stress predicted if the considered fibers were perfectly bonded. To correctly predict the overall tangent stiffness (and therefore the strain localization tensors), note that the tangent modulus determined from the constitutive equation of the partially debonded fibers accounts for the stress transfer ratio in the first step.

\section{Validation with finite element simulations}

\subsection{Introduction of a finite element model}

A 3D finite element model has been developed to compare the results obtained with the shear lag approach model. This FE model consist of a representative volume unit-cell (RUC) of cuboidal

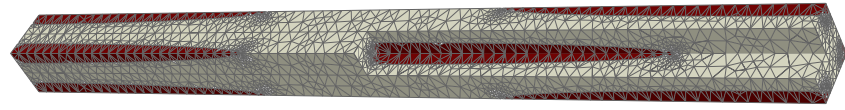

Fig. 5. FE models with cohesive elements on the interface between the matrix and short fiber modeled as ellipsoidal inclusions $\left(A R=22, V_{f}=0.18\right)$.

shape. It is composed of two prolate spheroidal inclusions representing the fibers (one centered in the RVE and the other on the limit of the RVE) and a matrix filling the remaining RVE (see Fig. 5). The geometry of the fibers is governed by an aspect ratio, named $A R$ (which is the major semi-axis length divided by the minor semi-axis length), and a fiber volume fraction, referred as $V_{f}$. These two parameters allow for the determination of the semi-axis length.

The geometry and the mesh are periodic, with periodic boundary conditions. The closest node from the center of the RVE is fully clamped. The loading is applied at the limit of the RVE using constraint drivers (see [36]). A constraint driver representing the average strain in one direction is applied along the tensile direction and the stresses are set to zero in all other directions. FE problems are solved using Abaqus Finite Element Analyses package.

To include the interfacial debonding mechanism, cohesive elements are inserted at the matrix-fiber interface. Cohesive elements are powerful numerical tools allowing the characterization of the fracture and fragmentation of various materials. The main principle of cohesive elements is that two initially adjacent surfaces are linked using a traction-separation relationship, which permits the simulation of a progressive debonding between these surfaces. These elements have been used to model different material responses such as the crack propagation in asphalt concrete beam [37] and the debonding in a fiber reinforced concrete beams [38]. A relevant review of traction-separation relationship for cohesive models has been reported by Park and Paulino [39], which focuses mainly on potential-based cohesive formulations.

\subsection{Selection of cohesive elements}

The selection of proper cohesive elements is mainly based on the following criteria: (i) ability to identify, at least partially, the traction-separation parameters based on the proposed model for interface damage, (ii) cohesive elements with zero initial volume and (iii) availability in the selected Finite Element Analyses package. For these three criteria, the element suite of Spring and Paulino [38] has been selected.

The selected elements use a potential-based traction-separation law. This guarantee the consistency of the constitutive relationship for any fracture under mixed-mode conditions, which is relevant to model the debonding of a fiber in 3D. Selected cohesive elements are based on the intrinsic PPR model developed by [23]. The potential of the constitutive law is given in Eq. (18). $\Phi_{n}$ and $\Phi_{t}$ are the normal and tangential fracture energies, $\Gamma_{n}$ and $\Gamma_{t}$ are constants related to the normal and tangential fracture energies, $\Delta_{n}$ and $\Delta_{t}$ are the normal and tangential opening traction displacements, $\delta_{n}$ and $\delta_{t}$ are normal and tangential final crack opening displacements, $\zeta_{n}$ and $\zeta_{t}$ are law shape parameters, $\lambda_{n}$ and $\lambda_{t}$ are the initial slope indicators, $N_{n}$ and $N_{t}$ are non-dimensional exponents related to $\zeta_{n}, \zeta_{t}, \lambda_{n}$ and $\lambda_{t}$. $<.>$ are the Macauley brackets.

$$
\begin{aligned}
\Pi\left(\Delta_{n}, \Delta_{t}\right)= & \min \left(\Phi_{n}, \Phi_{t}\right) \\
& +\left[\Gamma_{n}\left(1-\frac{\Delta_{n}}{\delta_{n}}\right)^{\zeta_{n}}\left(\frac{N_{n}}{\zeta_{n}}+\frac{\Delta_{n}}{\delta_{n}}\right)^{N_{n}}+<\Phi_{n}-\Phi_{t}>\right] \\
& \times\left[\Gamma_{t}\left(1-\frac{\left|\Delta_{t}\right|}{\delta_{t}}\right)^{\zeta_{t}}\left(\frac{N_{t}}{\zeta_{t}}+\frac{\left|\Delta_{t}\right|}{\delta_{t}}\right)^{N_{t}}+<\Phi_{t}-\Phi_{n}>\right]
\end{aligned}
$$


Inputs of these cohesive elements are therefore $\Phi_{n}, \Phi_{t}, \zeta_{n}, \zeta_{t}, \lambda_{n}$ and $\lambda_{t}$, as well as $\sigma_{\text {crit }}$ and $\tau_{\text {crit }}$ which are the normal and tangential cohesive strengths. Expressions of $N_{n}, N_{t}, \Gamma_{n}, \Gamma_{t}, \delta_{n}$ and $\delta_{t}$ are provided in Eq. (19).

$$
\left\{\begin{array}{l}
N_{n}=\frac{\zeta_{n}\left(\zeta_{n}-1\right) \lambda_{n}^{2}}{1-\zeta_{n} \lambda_{n}^{2}} \text { and } N_{t}=\frac{\zeta_{t}\left(\zeta_{t}-1\right) \lambda_{t}^{2}}{1-\zeta_{t} \lambda_{t}^{2}} \\
\Gamma_{n}=\left(-\Phi_{n}\right)^{<\Phi_{n}-\Phi_{t}>/\left(\Phi_{n}-\Phi_{t}\right)}\left(\frac{\zeta_{n}}{N_{n}}\right)^{N_{n}} \text { and } \Gamma_{t}=\left(-\Phi_{t}\right)^{<\Phi_{t}-\Phi_{n}>/\left(\Phi_{t}-\Phi_{n}\right)}\left(\frac{\zeta_{t}}{N_{t}}\right)^{N_{t}} \\
\delta_{n}=\frac{\Phi_{n}}{\sigma_{\max }} \zeta_{n} \lambda_{n}\left(1-\lambda_{n}\right)^{\zeta_{n}-1}\left(\frac{\zeta_{n}}{N_{n}}+1\right)\left(\frac{\zeta_{n}}{N_{n}} \lambda_{n}+1\right)^{N_{n}-1} \\
\delta_{t}=\frac{\Phi_{t}}{\tau_{\max }} \zeta_{t} \lambda_{t}\left(1-\lambda_{t}\right)^{\zeta_{t}-1}\left(\frac{\zeta_{t}}{N_{t}}+1\right)\left(\frac{\zeta_{t}}{N_{t}} \lambda_{t}+1\right)^{N_{t}-1}
\end{array}\right.
$$

Spring and Paulino [38] have implemented this cohesive law in user element subroutines (UEL) for three different types of 3D elements, which allows for easy use in Abaqus. The available elements are linear brick, linear tetrahedral and quadratic tetrahedral elements. Only linear brick elements have been used in this paper. To briefly describe the content of the algorithm behind this user element subroutine, five element states are available: the contact condition, the elastic condition, the softening condition, the unloading-reloading condition and complete failure. The contact condition is solved by a penalty stiffness approach. Under the softening condition, the traction force is given by the derivative of the potential (Eq. (20)). Under the unloading-reloading condition, the traction forces are reformulated assuming a linear elastic behavior law, without anelastic strain. Under the complete failure condition, the traction forces are null. The local traction vector is thus expressed as:

$t_{\text {local }}=\left(T_{n}, T_{t} \frac{\Delta_{1}}{\Delta_{t}}, T_{t} \frac{\Delta_{2}}{\Delta_{t}}\right)=\left(\frac{\partial \Pi}{\partial \Delta_{n}}, \frac{\partial \Pi}{\partial \Delta_{t}} \frac{\Delta_{1}}{\Delta_{t}}, \frac{\partial \Pi}{\partial \Delta_{t}} \frac{\Delta_{2}}{\Delta_{t}}\right)$

where $\Delta_{1}$ and $\Delta_{2}$ are the crack opening widths in the plane perpendicular to the normal direction and $\Delta_{t}=\sqrt{\Delta_{1}^{2}+\Delta_{2}^{2}}$. [38] can be consulted for more details.

Inspired by [39] and to provide an example of the tractionseparation typical behavior, the constitutive law is shown in Fig. 6. These curves result from a single element under traction (Mode I) and shear (Mode II). They represent the stress in the traction direction in the case of mode I and the maximum shear stress in the case of mode II. The parameters of the cohesive element model are $\Phi_{n}=100 \mathrm{~N} / \mathrm{m}, \Phi_{t}=200 \mathrm{~N} / \mathrm{m}, \quad \sigma_{\text {crit }}=4 \mathrm{MPa}, \tau_{\text {crit }}=$ $3 \mathrm{MPa}, \zeta_{n}=5, \zeta_{t}=1.6$ and $\lambda_{n}=\lambda_{t}=0.005$. During the tensile test, the stress increases linearly until it reaches $\sigma_{\text {crit }}$, then the softening behavior causes the stress to decrease from $\sigma_{\text {crit }}$ to zero. The curved shape during the softening phase is convex due to a $\zeta_{n}$ greater than 2. During the shear test, the stress increases linearly until it reaches

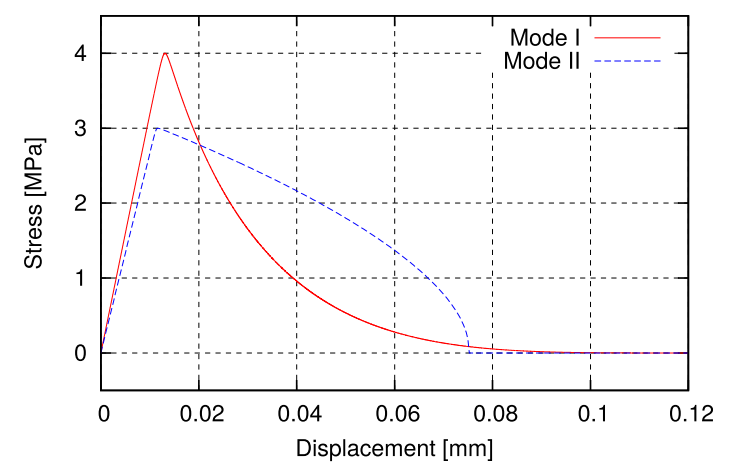

Fig. 6. Elementary tests of a cohesive element. Stress in the traction direction for mode I and maximum shear stress for mode II (from [39]). $\tau_{c r i t}$, then the softening behavior causes the stress decrease from $\tau_{\text {crit }}$ to zero. The curved shape during the softening phase is concave due to a $\zeta_{t}$ less than 2 .

\subsection{Geometry and properties of the SFRC in the FE model}

The PA66-GF30 is modeled by FE method using a SFRC to compare with the load transfer model (Fig. 5). This case is studied for various loading directions: $0^{\circ}$ (in the direction of the fiber), $15^{\circ}, 20^{\circ}, 45^{\circ}$ and $90^{\circ}$.

Geometric and material properties of the FE model with ellipsoidal inclusions are given in Table 1 . It should be noted that, based on the Tresca criterion, the tangential cohesive strengths $\tau_{\text {crit }}$ is set to be equal to half of the normal cohesive strengths $\sigma_{\text {crit }}$. All other parameters for cohesive elements are the same for all models: $\Phi_{n}=1.36 \mathrm{kN} / \mathrm{m}, \Phi_{t}=2.67 \mathrm{kN} / \mathrm{m}, \zeta_{n}=\zeta_{t}=5$ and $\lambda_{n}=\lambda_{t}=0.005$. $\Phi_{n}$ and $\Phi_{t}$ are given by [40]. $\zeta_{n}$ and $\zeta_{t}$ are chosen to obtain a quick total failure condition when softening appears. $\lambda_{n}$ and $\lambda_{t}$ are chosen to obtain the lowest value of the ratio of the critical crack opening width to the final crack opening width. Conversely to a crack, the interface debonding is considered to occur with a low opening displacement. C3D4 elements were used to model the matrix $(54,588$ elements) and the fibers (239,860 elements), and pentahedric elements were used at the interfaces $(13,856$ elements).

\subsection{Comparison with the proposed model}

Von Mises stresses resulting from the FE simulation of the PA66-GF30 in the case of loading at $0^{\circ}$ (in the direction of the fiber) are shown on Fig. 7. For the sake of clarity, the displacement scale factor is set to 10 and only half of the RVE is shown. The debonded area at the matrix-fiber interface can be seen clearly. As presented in the bibliography, in the case of short fiber reinforced composite material, debonding at the matrix-fiber interface often appears on top of the fibers. Within this case study, debonding starts where the tensile stresses at the interface are maximum and spreads along the reinforcements. The interfacial damage reduces the stress level at the fiber extremities while the Von Mises stress in the middle of the fiber is still high. These observations are in agreement with the main hypothesis of a shear-lag approach, i.e. the load transfer driven by the shear stress.

The comparison, in terms of effective stress-strain responses, between the FE model with cohesive elements and the new model is illustrated in Fig. 8. The FEA model response has been utilized to identify the parameters of the newly proposed method. The method applied for identification is a hybrid optimisation algorithm associating a genetic algorithm optimised with a Levenberg-Marquardt method [41]. The identified parameters of the damage evolution law at the interface used for the comparison of results are $\eta=1.61, \quad \gamma=2.084, \quad \kappa=0.037, \quad \omega=2.962$ and $\alpha=0.317$.

Since it has been shown that debonding can be captured properly with the FEA unit model, this model is further utilized to study the capabilities of the proposed approach. Considering the PA66GF30 composite material, five orientations have been simulated using the FE model with cohesive elements. The results are shown in Fig. 8. The overall non-linear response of five composites reinforced with fibers in different directions are all captured with accuracy. Note that the high strain reached for purely elastic materials

Table 1

Material and geometric parameters of the composite material PA66-GF30.

\begin{tabular}{llllllll}
\hline & $E_{0}(\mathrm{GPa})$ & $v_{0}$ & $E_{1}(\mathrm{GPa})$ & $v_{1}$ & $V_{f}$ & $A R$ & $\sigma_{\text {crit }}(\mathrm{MPa})$ \\
\hline \multirow{2}{*}{ PA66-GF30 } & 3.6 & 0.36 & 72 & 0.26 & 0.18 & 22 & 118 \\
\hline
\end{tabular}




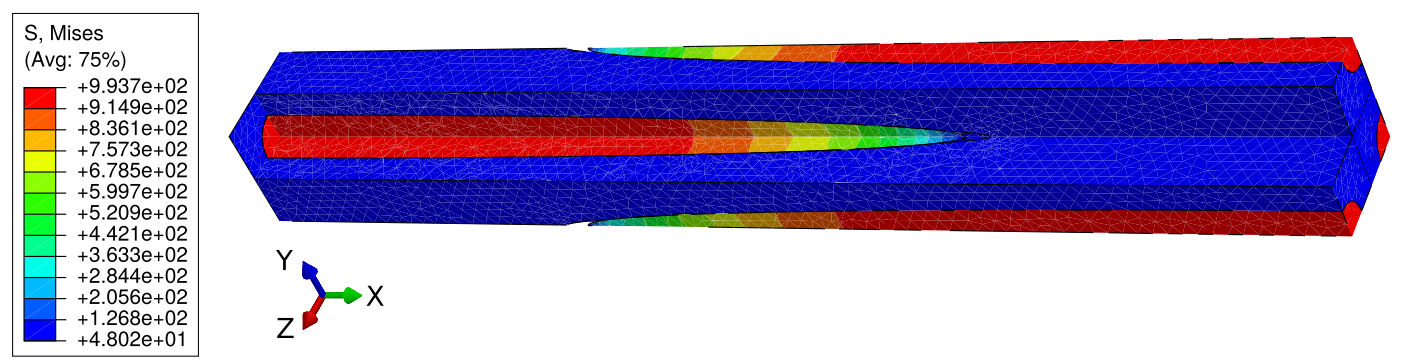

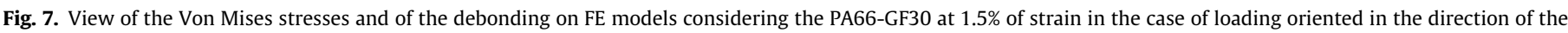
fiber $\left(0^{\circ}\right)$. Strain is zoomed X10. Only half of the RVE is represented.

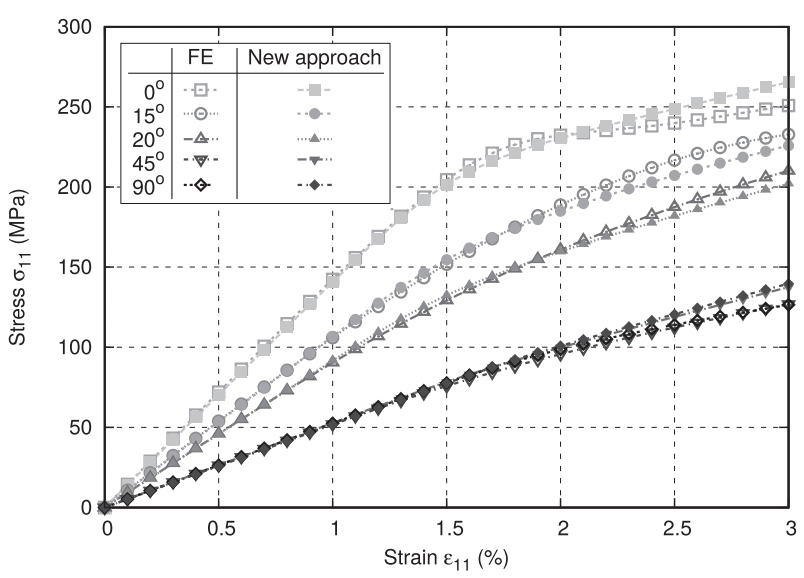

Fig. 8. Comparison of strain-stress curves between FE model with cohesive elements and the present model for various loading directions for PA66-GF30 composite.

correspond to a severe debonding of the fibers. Debonding initiations have been well captured.

Since only one set of parameters has been used to capture the effect of all fiber orientations, it clearly shows the influence of the local curvature $C(\boldsymbol{x})$ introduced in the probability function (4). In the case of injected composites with a complex microstructure and multiple orientation of fibers, such capabilities will be of the utmost importance since they will facilitate an inverse identification approach to determine the characteristic parameters of damage evolution.

\section{Validity limit of the new approach}

In this section, the capability limits of the proposed model are evaluated. The inspired shear lag solution has been justified for the case of ellispoidal inclusions, since it has been shown experimentally that debonding primarily occurs at the fiber ends. However, many composite materials contain spherical inclusions, which can be seen as a limiting case of ellipsoidal inclusions. Several experimental results and numerical models are available in the literature to further validate the proposed model for this specific case.

Comparisons are provided and discussed. To this end, FE results and those obtained by the proposed approach are compared to experimental and numerical results from the literature. For each simulation performed with the new model, the parameters of the damage law $(\eta, \gamma, \kappa$ and $\omega)$ are identified by the same reverse engineering algorithm utilized in the previous section. In this case, however, the parameter $\alpha$ is of no interest as the curvature of a sphere is constant, and is therefore not present in this section.

\subsection{A weakened interface approach}

The developed approach is now compared with a similar work based on imperfect interfaces from [26]. They modeled the effect of slightly weakened interfaces on the overall elastic properties of composite materials reinforced by spherical particles. Their micromechanical model is based on the work of [42], who developed a solution for the Eshelby tensor considering an elastic inclusion with a slightly weakened interface. A phase of a slightly weakened (debonded) fiber phase is considered. The volume fraction of this new phase is determined using an evolution equation based on the average internal stresses of the particles and two Weibull parameters. The effective elastic properties of the damaged composite are then estimated using a three-phase particulate model.

In a subsequent paper [43], they have developed a multi-level damage model. The evolution of damage (taken from [43]) is depicted in Fig. 9: (a) at the initial stage, the composite consists of 2 phases (matrix and particles); (b) a third phase is added for a slightly damaged composite material; (c) a fourth phase is added for severely damaged interfaces - now, four phases are homogenized, including two levels of matrix-particle interface damage; (d) a last phase is added to consider voids resulting from debonded fibers. Each damage mechanism is driven by a Weibull-like damage law. Each particle has its own damage parameter and its own evolution law.

The present section consists of a comparison of the proposed model between:

1. A theoretical case study comparing the weakened interface approach [26] and a partial debonding interface approach developed by Ju and Lee [44].

2. Experimental data acquired by Zhou et al. [24] and the weakened interface approach of [26].

3. Experimental data acquired by Sorensen [25] and the multilevel damage model of [43].

\subsection{Comparison with the weakened interface approach}

Lee and Pyo [26] have compared a partially debonded interface approach developed by Ju and Lee [44] to the experimental data acquired by Zhou et al. [24]. They have further compared their multi-level damage model with the experimental data acquired by Sorensen [25]. The properties of these composite materials are given in Table 2. These results are compared with the proposed model. All identified values for these simulations are sumarized in Table 3. According to the Tresca criterion, $\tau_{\text {crit }}$ is set at half the value of $\sigma_{\text {crit }}$, which is given in the literature.

Fig. 10 shows the comparison between the weakened interface approach, a partially debonded interface model developed by Ju and Lee [44] and the new approach. Note that the parameters of 
a

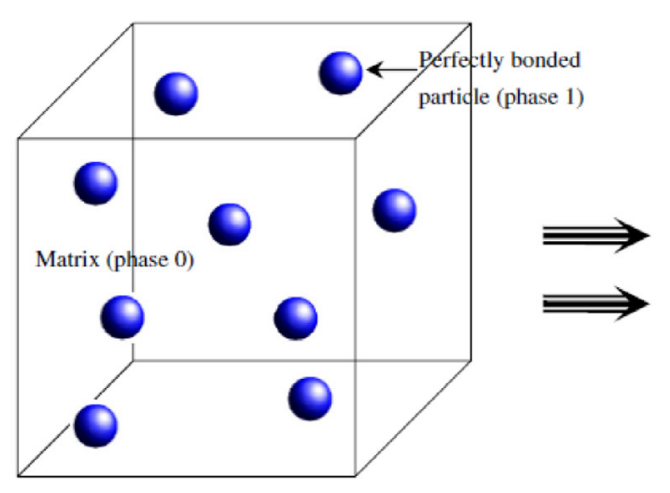

b
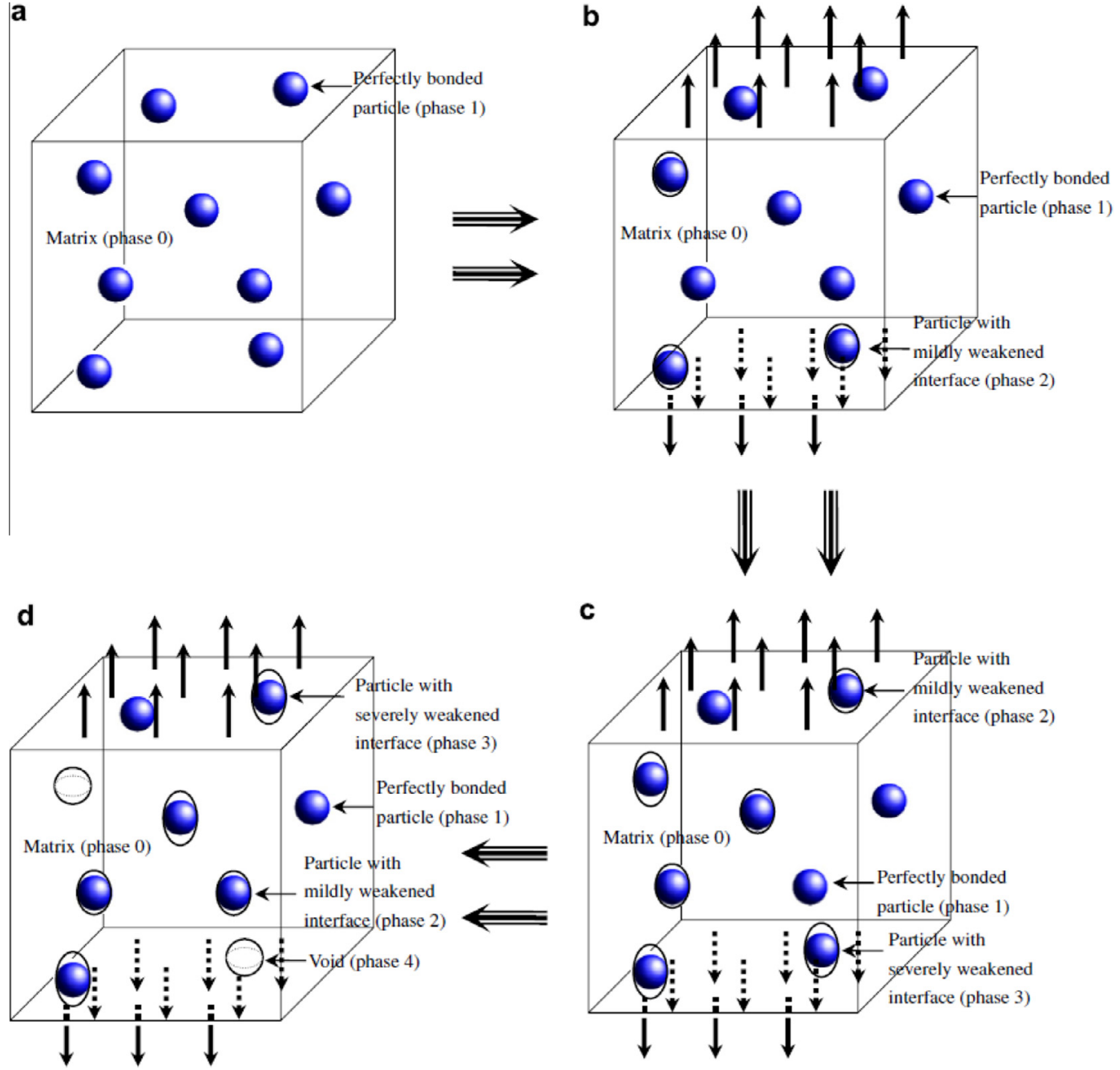

C

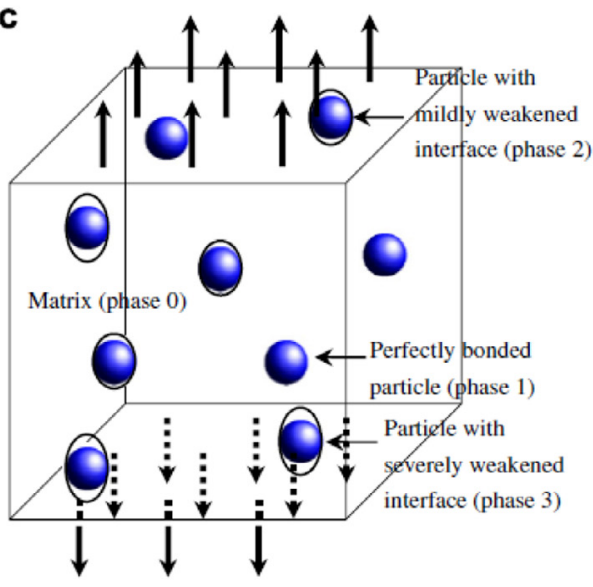

Fig. 9. Evolution of a representative volume of a spherical reinforced composite as a function of successive damage states of matrix-fiber interfaces (from [43]).

Table 2

Material parameters of analyzed composite materials in [26,43].

\begin{tabular}{|c|c|c|c|}
\hline \multirow[t]{2}{*}{ Property } & Material 1 & Material 2 & Material 3 \\
\hline & $\begin{array}{l}\text { 6061-T6 Al matrix } \\
\text { SiC reinforc. }\end{array}$ & $\begin{array}{l}\mathrm{Mg} \text { - } \mathrm{Al} \text { matrix } \\
\mathrm{Al}_{2} \mathrm{O}_{3} \text { reinforc. }\end{array}$ & $\begin{array}{l}\text { CAS matrix } \\
\text { SiC reinforc. }\end{array}$ \\
\hline Young's modulus of the matrix $\left(E_{0}\right)$ & $68.3 \mathrm{GPa}$ & $73 \mathrm{GPa}$ & $98 \mathrm{GPa}$ \\
\hline Poisson's ratio of the matrix $\left(v_{0}\right)$ & 0.33 & 0.33 & 0.3 \\
\hline Young's modulus of reinforc. $\left(E_{1}\right)$ & $490 \mathrm{GPa}$ & $400 \mathrm{GPa}$ & $200 \mathrm{GPa}$ \\
\hline Poisson's ratio of reinforc. $\left(v_{1}\right)$ & 0.17 & 0.24 & 0.15 \\
\hline Volume fraction of reinforc. $\left(V_{f}\right)$ & 0.2 & 0.48 & 0.35 \\
\hline Critical stress at the interface $\left(\sigma_{\text {crit }}\right)$ & $250 \mathrm{MPa}$ & $292 \mathrm{MPa}$ & $450 \mathrm{MPa}$ \\
\hline
\end{tabular}

Table 3

Parameters of the damage evolution law at the interface used for comparison of results coming from Ju and Lee [44] (6061-T6 Al matrix SiC reinforc.), Zhou et al. [24] ( $\mathrm{Mg}$ - $\mathrm{Al}$ matrix $\mathrm{Al}_{2} \mathrm{O}_{3}$ reinforc.) and Sorensen [25] (CAS matrix SiC reinforc.).

\begin{tabular}{lllll}
\hline Material & \multicolumn{4}{c}{ Parameters } \\
\cline { 2 - 5 } & $\eta$ & $\gamma$ & $\kappa$ & $\omega$ \\
\hline 6061-T6 $\mathrm{Al}$ matrix $\mathrm{SiC}$ reinforc. & 3.2 & 1.23 & 0.001 & -2.95 \\
$\mathrm{Mg}-\mathrm{Al}$ matrix $\mathrm{Al}_{2} \mathrm{O}_{3}$ reinforc. & 3.1 & 1.19 & 0.01 & 2.99 \\
$\mathrm{CAS}$ matrix $\mathrm{SiC}$ reinforc. & 2.8 & 0.74 & 0.036 & -1.11 \\
\hline
\end{tabular}

the new approach were identified from the results of the weakened interface model of [43], which is considered as the most accurate. In this figure, two asymptotical behaviors are added: an undamaged material (with perfect bonding) acting as a purely elastic material, and a porous equivalent material, replacing the debonded inclusions with voids. The homogenization of the effective behavior is computed with the Mori-Tanaka micromechanical method. $\mathrm{Ju}$ and Lee's results quickly converge to the porous equivalent material while Lee and Pyo's model, despite being able to get the initial debonding converge to another asymptote between both 


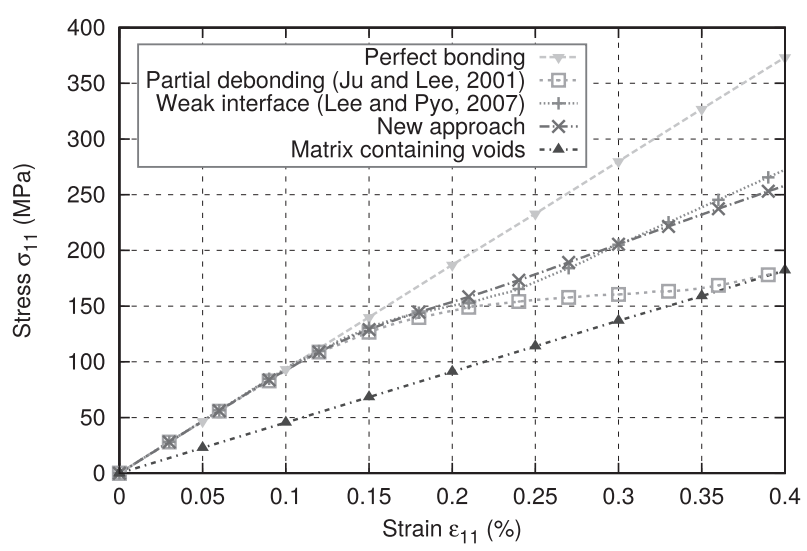

Fig. 10. Comparison of the predicted overall uniaxial responses of 6061-T6 aluminum alloy matrix/silicon-carbide particle composites having (i) a perfect interface (elastic), (ii) the partial debonding model by Ju and Lee [44], (iii) the weakened interface according to the damage model by Lee and Pyo [26], (iv) the present approach based on the modified SLM and (v) the matrix containing voids.

extreme behaviors. In this case, the proposed model is able to reproduce the initial debonding of the two previous approaches, as well as fit closely to the evolution of the weakened interface model.

To further evaluate the model capabilities, comparisons with experimental data obtained by Zhou et al. [24] are provided. The considered material is material 2 in Table 2. Here, the initial debonding determined by the proposed approach fits perfectly with the experimental data (Fig. 11) and and slightly better than the weak interface model, which was already very accurate.

A last comparison is made with [25] where the related material is material 3 in Table 2. In this case, Lee and Pyo have used the more complex model (Fig. 9). The comparison between the different approaches is shown in Fig. 12. Although the initial effective debonding stress is underestimated for both models, the asymptotical behavior of the experimental data is captured better by the new approach than by Lee and Pyo's model. The developed approach is able to fit the non-linear evolution of the material response with great accuracy.

Beside the ability to capture the non-linear response of composite materials containing debonded interfaces, one of the major advantage of this new method is that it requires only four parameters in addition to the constitutive parameters of the phases and the critical stress for debonding. Actually, the proposed method

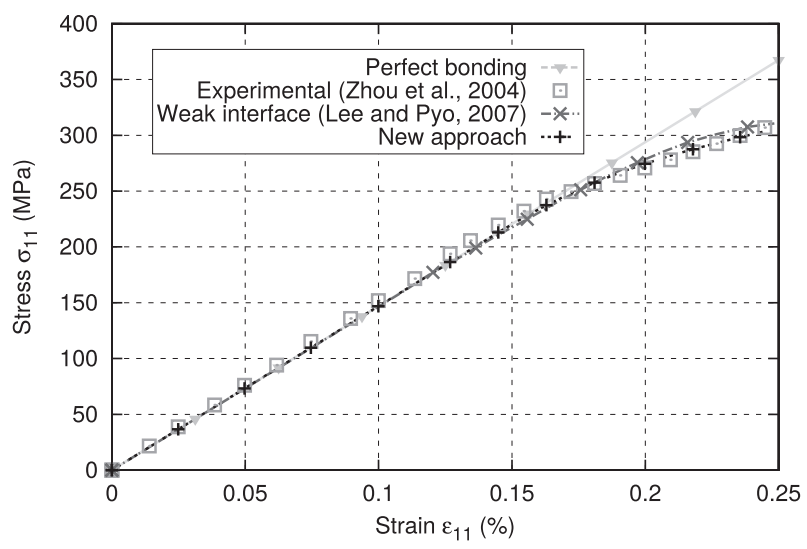

Fig. 11. Comparison of predicted overall uniaxial responses of $\mathrm{Mg}$ - $\mathrm{Al}$ matrix with $\mathrm{Al}_{2} \mathrm{O}_{3}$ particulate composites between experimental data from [24], the weakened interface approach [26] and the present model.

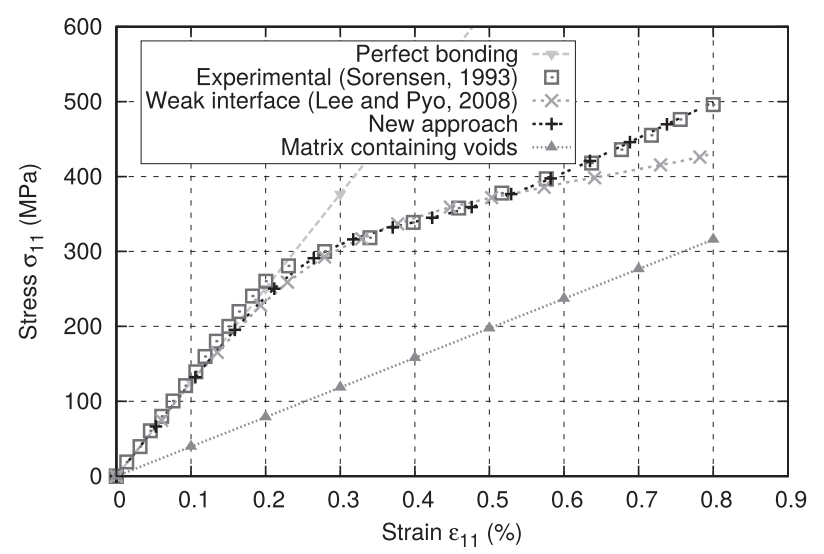

Fig. 12. Comparison of predicted overall uniaxial responses of CAS matrix with $\mathrm{SiC}$ particulate composites between experimental data from [25], the weakened interface approach [43] and the present model.

is based on a two phases approach whereas five phases are utilized for Pyo and Lee's model. It should be noted that more than two phases can make the computation of the effective behavior more difficult when utilizing micro mechanical methods, as shown by Benveniste et al. [45]. It should also be noted that the case of spherical inclusions is actually a limiting case for this method, since the model has been primarily developed for short fiber composites.

To further validate the proposed approach, and at the same time to gain confidence in the numerical validation of the model in Section 4, comparisons are provided for the limiting case of spherical inclusions with numerical simulations obtained from RUC models containing cohesive elements and experimental results from [24].

\subsection{Capability limit with FE model}

A RUC model containing two spherical reinforcements is defined in a similar way to the RUC model presented in Section 3. Simulations are performed for an $\mathrm{Al}$ matrix with $\mathrm{SiC}$ reinforcements and a $\mathrm{Mg}$ - $\mathrm{Al}$ matrix with $\mathrm{Al}_{2} \mathrm{O}_{3}$ reinforcements that correspond to material 1 and 2 from Table 2. Geometric properties of the FE model with spherical inclusions are given in Table 1 . The number of elements and element types for each configuration are summarised in Table 4. The mesh of the material 1 ( $A l$ matrix with $\mathrm{SiC}$ reinforcement) is shown in Fig. 13a.

Fig. 13b shows a contour plot of Von Mises stresses for the case of an $\mathrm{Al}$ matrix and $\mathrm{SiC}$ reinforcement. The displacement scale factor is set to 10 to emphasize the debonded regions.

The comparison between the FE model with cohesive elements and the proposed approach is illustrated in Fig. 14, in terms of effective stress-strain responses for the case of an $\mathrm{Al}$ matrix and $\mathrm{SiC}$ reinforcement. The FE model response has been utilized to identify the parameters of the newly proposed method. Identified parameters are summarized in Table 5. A good correlation is observed for both materials. With the shear lag based method, an inflection point appears during the debonding, leading to potential

Table 4

Type and number of elements in FE models.

\begin{tabular}{lllll}
\hline Element type & $\begin{array}{l}\text { Linear } \\
\text { tetrahedral } \\
\text { Location }\end{array}$ & \multicolumn{2}{c}{ C3D4 } & Total \\
\cline { 3 - 4 } & Interface & Matrix & Fibers & \\
\hline $\begin{array}{c}A l \text { Matrix with } \mathrm{SiC} \\
\quad \text { reinforc. }\end{array}$ & 8352 & 98,536 & 313,766 & 420,654 \\
$\begin{array}{c}M g-A l \text { matrix } \mathrm{Al}_{2} \mathrm{O}_{3} \\
\quad \text { reinforc. }\end{array}$ & 8776 & 150,507 & 145,455 & 296,838 \\
\hline
\end{tabular}




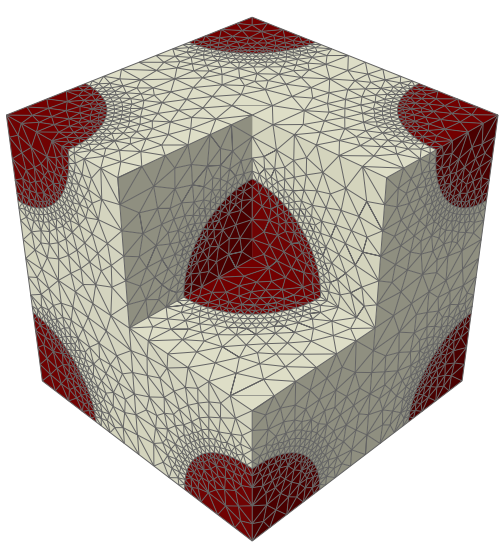

(a) Mesh

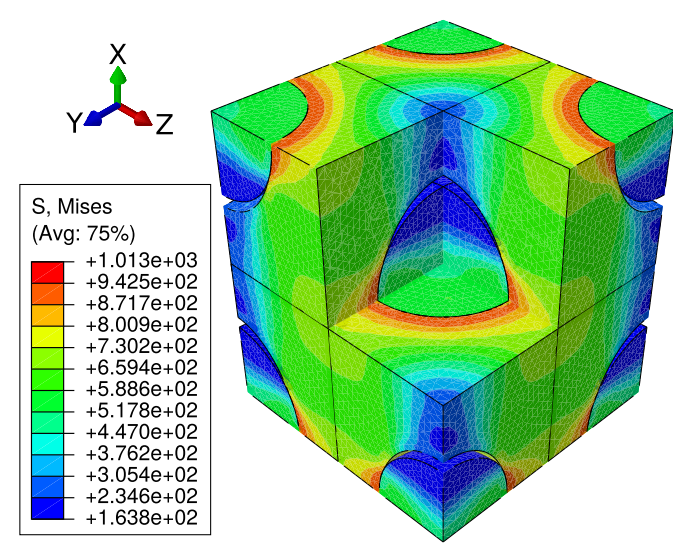

(b) Strain (zoomed X10) and Von Mises stresses at $1 \%$ of overall strain

Fig. 13. FE models including cohesive elements with spherical inclusions $\left(A R=1, V_{f}=0.2\right)$.

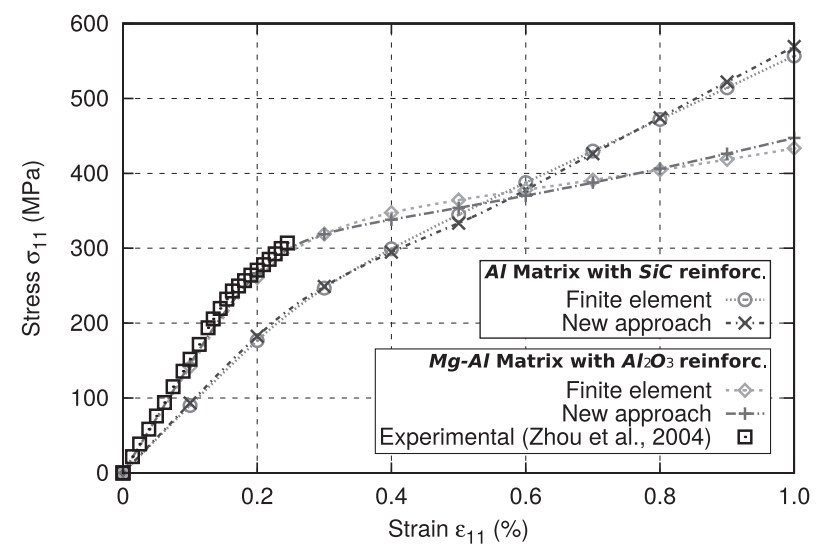

Fig. 14. Comparison of predicted overall uniaxial responses of $\mathrm{Al}$ matrix with $\mathrm{SiC}$ particulate composites (the FE model with cohesive elements and the present model based on modified SLM) and of $\mathrm{Mg}$ - $\mathrm{Al}$ matrix with $\mathrm{Al}_{2} \mathrm{O}_{3}$ particulate composites (experimental data from [24], the FE model with cohesive elements and the present model based on modified SLM.

Table 5

Parameters of the damage evolution law at the interface used for comparison of results from the FE model with cohesive interface.

\begin{tabular}{lllll}
\hline Materials & \multicolumn{4}{l}{ Parameters } \\
\cline { 2 - 5 } & $\eta$ & $\gamma$ & $\kappa$ & $\omega$ \\
\hline $\mathrm{Al}$ Matrix with $\mathrm{SiC}$ reinforc. & 2.9 & 1.02 & 0.015 & -0.23 \\
$\mathrm{Mg}$ - $\mathrm{Al}$ matrix $\mathrm{Al}_{2} \mathrm{O}_{3}$ reinforc. & 5.01 & 0.89 & 0.06 & -2.96 \\
\hline
\end{tabular}

errors for larger strains. According to the the second material ( $\mathrm{Mg}$ - $\mathrm{Al}$ matrix with $\mathrm{Al}_{2} \mathrm{O}_{3}$ reinforcement), the results are compared with experimental data extracted from [24]. The FE model and the proposed approach are both close to the experimental data. This ensures the validity of the developed FE investigation the regarding experimental data since the damage evolution is well captured.

\section{Conclusions}

A new original approach based on the shear lag model has been proposed in order to improve the simulation of short fiber reinforced composites. A probabilistic damage evolution equation based on a quadratic failure criterion has been implemented. Particular attention has been devoted to the probabilistic nature of such an evolution equation, in order to represent the debonding of a composite material with a large number of fibers. Also, the effect of the orientation of the fiber with respect to the applied stress is considered, using the determination of the local curvature of the fiber-matrix interface when damage appears. Based on the shear lag model, a new load transfer ratio has been proposed with the advantage of being easily implemented in incremental micromechanical approaches. If the developed approach is primarily developed for short fiber composites, it can be adapted to a large variety of composites with different microstructures and reinforcements.

Several comparisons with numerical reference solutions, experimental data and micromechanical models with weakened interfaces have been made in order to study the capabilities of the proposed model, as well as to provide numerical and experimental validation.

A comparison with a Numerical Reference Solution based on FEA with cohesive elements that represent the fiber-matrix interfaces was performed to show the capabilities of the approach to simulate the behavior of short-fiber composites. A good correlation is observed between the FE model, the new approach and the experimental results.

Further comparisons with results relative to a spherical inclusion reinforced composite have shown that the model is able to accurately predict the evolution of the effective behavior of the composite while it experiences significant damage, although spherical inclusions are considered a limiting case of the proposed approach.

Overall, for each comparison, the new model has proved its ability to reproduce the behavior of a composite material subjected to damage at the matrix reinforcement interface whether considering experimental results or numerical reference solutions. Furthermore, the proposed load transfer model is based on the shear lag model and, while several hypotheses about the shape of the reinforcements are necessary, it does not require additional parameters. Another advantage of this method is that only two phases are considered, which simplifies the homogenization step. Considering the shear lag model, a strong assumption is that debonding starts at the fiber ends, whereas the damage evolution law is actually able to provide the localization of maximal damage. Such evaluation of the precise localization of the debonding area may lead to new load transfer models that will be able to capture the debonding evolution, including its initial starting position.

Some parameters of the proposed damage law have to be identified by a reverse engineering procedure from the macroscopic 
behavior since it is difficult to characterize the debonding on a single fiber-matrix interface. In addition, in the present work, the interfacial damage law is computed along the entire ellipsoid surface by varying the angular step. Adopting small steps yields time consuming simulations, but the computations still remain faster (roughly 100 times) than those based on cohesive FE analyses. For structural simulations using the developed approach, an optimized procedure will required the identification of critical damage points.

\section{Acknowledgments}

The support of the FUI project Durafip, driven by Dr. Gilles Robert of Solvay Engineering Plastics, is warmly acknowledged.

\section{References}

[1] Arif M, Saintier N, Meraghni F, Fitoussi J, Chemisky Y, Robert G. Multiscale fatigue damage characterization in short glass fiber reinforced polyamide- 66 . Composites Part B 2014;61:55-65.

[2] Sato N, Kurauchi T, Sato S, Kamigaito O. Microfailure behaviour of randomly dispersed short fibre reinforced thermoplastic composites obtained by direct SEM observation. J Mater Sci 1991;26(14):3891-8.

[3] Horst JJ, Spoormaker JL. Fatigue fracture mechanisms and fractography of short-glassfibre-reinforced polyamide 6. J Mater Sci 1997;32(14):3641-51.

[4] Arif M, Meraghni F, Chemisky Y, Despringre N, Robert G. In situ damage mechanisms investigation of PA66/GF30 composite: effect of relative humidity. Composites Part B 2014;58:487-95.

[5] Bernasconi A, Davoli P, Basile A, Filippi A. Effect of fibre orientation on the fatigue behaviour of a short glass fibre reinforced polyamide-6. Int J Fatigue 2007;29(2):199-208.

[6] Mortazavian S, Fatemi A. Fatigue behavior and modeling of short fiber reinforced polymer composites: a literature review. Int J Fatigue 2015;70:297-321.

[7] Tucker III CL, Liang E. Stiffness predictions for unidirectional short-fiber composites: review and evaluation. Compos Sci Technol 1999;59(5):655-71.

[8] Desrumaux F, Meraghni F, Benzeggagh ML. Generalised mori-tanaka scheme to model anisotropic damage using numerical eshelby tensor. J Compos Mater 2001;35(7):603-24.

[9] Meraghni F, Desrumaux F, Benzeggagh M. Implementation of a constitutive micromechanical model for damage analysis in glass mat reinforced composite structures. Compos Sci Technol 2002;62(16):2087-97.

[10] Christensen R, Lo K. Solutions for effective shear properties in three phase sphere and cylinder models. J Mech Phys Solids 1979;27(4):315-30.

[11] Hashin Z. Thermoelastic properties of fiber composites with imperfect interface. Mech Mater 1990;8(4):333-48.

[12] Hashin Z. Thin interphase/imperfect interface in elasticity with application to coated fiber composites. J Mech Phys Solids 2002;50(12):2509-37.

[13] Benveniste Y, Miloh T. Imperfect soft and stiff interfaces in two-dimensional elasticity. Mech Mater 2001;33(6):309-23.

[14] Hazanov S. On micromechanics of imperfect interfaces in heterogeneous bodies smaller than the representative volume. Int J Eng Sci 1999;37 (7):847-61.

[15] Eshelby JD. The determination of the elastic field of an ellipsoidal inclusion, and related problems. Proc R Soc London, A 1957;241(1226):376-96.

[16] Zhong Z, Meguid SA. On the elastic field of a shpherical inhomogeneity with an imperfectly bonded interface. J Elast 1997;46(2):91-113.

[17] Cox HL. The elasticity and strength of paper and other fibrous materials. Br J Appl Phys 1952:3(3):72.

[18] Jain A, Abdin Y, Paepegem WV, Verpoest I, Lomov SV. Effective anisotropic stiffness of inclusions with debonded interface for Eshelby-based models. Compos Struct 2015;131:692-706.

[19] Clyne TW, Withers PJ. An introduction to metal matrix composites. Cambridge University Press; 1993.
20] Nairn JA. On the use of shear-lag methods for analysis of stress transfer in unidirectional composites. Mech Mater 1997;26(2):63-80.

[21] Jiang X, Gao Q. Stress-transfer analysis for fibre/matrix interfaces in shortfibre-reinforced composites. Compos Sci Technol 2001;61(10):1359-66.

[22] Lagoudas D, Gavazzi A, Nigam H. Elastoplastic behavior of metal matrix composites based on incremental plasticity and the mori-tanaka averaging scheme. Comput Mech 1991;8(3):193-203.

[23] Park K, Paulino GH, Roesler J. Cohesive fracture model for functionally graded fiber reinforced concrete. Cem Concr Res 2010;40(6):956-65.

[24] Zhou C, Yang W, Fang D. Mesofracture of metal matrix composites reinforced by particles of large volume fraction. Theor Appl Fract Mech 2004;41(1 3):311-26.

[25] Sorensen BF. Effect of fibre roughness on the overall stress-transverse strain response of ceramic composites. Scr Metall Mater 1993;28(4):435-9.

[26] Lee H, Pyo S. Micromechanics-based elastic damage modeling of particulate composites with weakened interfaces. Int J Solids Struct 2007:44(2526):8390-406

[27] Cosmi F, Bernasconi A. Micro-ct investigation on fatigue damage evolution in short fibre reinforced polymers. Compos Sci Technol 2013:79:70-6.

[28] Jendli Z, Meraghni F, Fitoussi J, Baptiste D. Multi-scales modelling of dynamic behaviour for discontinuous fibre SMC composites. Compos Sci Techno 2009;69(1):97-103.

[29] Meraghni F, Blakeman C, Benzeggagh M. Effect of interfacial decohesion on stiffness reduction in a random discontinuous-fibre composite containing matrix microcracks. Compos Sci Technol 1996;56(5):541-55.

[30] L.N. McCartney, Stress transfer mechanics for multiple perfectly bonded concentric cylinder models of unidirectional composites, NPL Report $\operatorname{DMM}(\mathrm{A})$ 129.

[31] Mura T. Micromechanics of defects in solids. Kluwer Academic Publishers: 1987.

[32] Mori T, Tanaka K. Average stress in matrix and average elastic energy of materials with misfitting inclusions. Acta Metall 1973;21(5):571-4.

[33] Benveniste Y. A new approach to the application of Mori-Tanaka's theory in composite materials. Mech Mater 1987;6(2):147-57.

[34] Desrumaux F, Meraghni F, Benzeggagh ML. Micromechanical modelling coupled to a reliability approach for damage evolution prediction in composite materials. Appl Compos Mater 2000;7(4):231-50.

[35] Gavazzi AC, Lagoudas DC. On the numerical evaluation of Eshelby's tensor and its application to elastoplastic fibrous composites. Comput Mech 1990;7:13-9.

[36] Li S, Wongsto A. Unit cells for micromechanical analyses of particle-reinforced composites. Mech Mater 2004;36(7):543-72.

[37] Song SH, Paulino GH, Buttlar WG. A bilinear cohesive zone model tailored for fracture of asphalt concrete considering viscoelastic bulk material. Eng Fract Mech 2006:73(18):2829-48.

[38] Spring DW, Paulino GH. A growing library of three-dimensional cohesive elements for use in ABAQUS. Eng Fract Mech 2014;126:190-216.

[39] Park K, Paulino GH. Cohesive zone models: a critical review of tractionseparation relationships across fracture surfaces. Appl Mech Rev 2013;64 (6):60802

[40] Starke C, Beckert W, Lauke LB. Charakterisierung des delaminationsverhaltens von schichtverbunden unter mode i und mode iibelastungen. Materialwiss Werkstofftech 1996;27:080-9.

[41] Chemisky Y, Meraghni F, Bourgeois N, Cornell S, Echchorfi R, Patoor E. Analysis of the deformation paths and thermomechanical parameter identification of a shape memory alloy using digital image correlation over heterogeneous tests. Int J Mech Sci 2015;9697:13-24.

[42] Qu J. Eshelby tensor for an elastic inclusion with slightly weakened interface. J Appl Mech 1993;60(4):1048-50.

[43] Lee H, Pyo S. Multi-level modeling of effective elastic behavior and progressive weakened interface in particulate composites. Compos Sci Technol 2008;68 (2):387-97.

[44] Ju J, Lee H. A micromechanical damage model for effective elastoplastic behavior of partially debonded ductile matrix composites. Int J Solids Struct 2001;38(36-37):6307-32.

[45] Benveniste Y, Dvorak G, Chen T. On diagonal and elastic symmetry of the approximate effective stiffness tensor of heterogeneous media. J Mech Phys Solids 1991;39(7):927-46. 